\title{
On Kissing Numbers in Dimensions 32 to 128
}

\author{
Yves Edel \\ Mathematisches Institut der Universität \\ Im Neuenheimer Feld 288 \\ 69120 Heidelberg, Germany \\ E. M. Rains and N. J. A. Sloane \\ AT\&T Labs-Research \\ 180 Park Avenue \\ Florham Park, NJ 07932-0971, USA
}

Submitted: April 9, 1998; Accepted: April 13, 1998

\begin{abstract}
An elementary construction using binary codes gives new record kissing numbers in dimensions from 32 to 128.
\end{abstract}

\section{Introduction}

Let $\tau_{n}$ denote the maximal kissing number in dimension $n$, that is, the greatest number of $n$-dimensional spheres that can touch another sphere of the same size. Although asymptotic bounds on $\tau_{n}$ are known [5], little is known about explicit constructions, especially for $n>32$. Up to now the best explicit constructions have come from lattice packings. The kissing number $\tau$ of the Barnes-Wall lattice ${ }^{1} B W_{n}$ in dimension $n=2^{m}$ is $\prod_{i=1}^{m}\left(2^{i}+2\right)$, although for $m \geq 5$ this is weak $(146,880,9,694,080$ and 1,260,230,400 in dimensions 32,64 and 128, for example). In contrast, Quebbemann's lattice $Q_{32}$ [14], [5, Chap. 8] has $\tau=261,120$.

In recent years the kissing numbers of a few other lattices in dimensions $>32$ have been determined. Nebe [10] shows that the Mordell-Weil lattice $M W_{44}$ has $\tau=2,708,112$. Nebe [11] shows that a 64-dimensional lattice constructed in [10] is extremal 3-modular, and so by modular form theory has $\tau=138,458,880$. Bachoc and Nebe [1] give an 80-dimensional lattice with $\tau=1,250,172,000$. Elkies [6] calculated the kissing number of his lattice $M W_{128}$ : it is $218,044,170,240$, over 170 times that of $B W_{128}$.

In the present note, we show that an elementary construction using binary codes gives better values than all of these. However, our packings are just local arrangements of spheres

\footnotetext{
${ }^{1}$ The subscript gives the dimension.
} 
around the origin: we do not know if they can be modified to produce dense infinite packings.

\section{The construction}

Let $\mathcal{C}(n, d)$ (resp. $\mathcal{C}(n, d, w))$ denote a set of binary vectors of length $n$ and Hamming distance $\geq d$ apart (resp. and with constant weight $w$ ). The maximal size of such a set is denoted by $A(n, d)$ (resp. $A(n, d, w))$ [2], [9].

One way to achieve the kissing number $\tau_{8}=240$ in eight dimensions is to take as centers of spheres the vectors of shape $\pm 1^{8}$, with a unique support (a code $\mathcal{C}(8,8,8) !$ ) and signs taken from a $\mathcal{C}(8,2)$, together with the vectors of shape $\pm 2^{2} 0^{6}$, where the supports are taken from a $\mathcal{C}(8,2,2)$ and the signs from a $\mathcal{C}(2,1)$. Taking all these codes to be as large as possible, we obtain a total of

$$
A(8,8,8) A(8,2)+A(8,2,2) A(2,1)=1 \cdot 2^{7}+\left(\begin{array}{l}
8 \\
2
\end{array}\right) 2^{2}=240
$$

spheres touching the sphere at the origin.

Our construction generalizes this as follows. For a given dimension $n$, we choose a sequence of support sizes $n_{0}, n_{1}, \ldots, n_{\mu}$ satisfying

$$
n \geq n_{0} \geq 4 n_{1} \geq 4^{2} n_{2} \geq \cdots \geq 4^{\mu} n_{\mu} \geq 1 .
$$

The $\nu^{\text {th }}$ set of centers that we use, for $0 \leq \nu \leq \mu$, consists of vectors of shape $\pm a_{\nu}^{n_{\nu}} 0^{n-n_{\nu}}$, where

$a_{\nu}=\sqrt{n_{0} / n_{\nu}}$, the supports are taken from a $\mathcal{C}\left(n, n_{\nu}, n_{\nu}\right)$ and the signs from a $\mathcal{C}\left(n_{\nu},\left\lceil\frac{n_{\nu}}{4}\right\rceil\right)$. With optimal codes, the total number of vectors is

$$
\sum_{\nu=0}^{\mu} A\left(n, n_{\nu}, n_{\nu}\right) A\left(n_{\nu},\left\lceil\frac{n_{\nu}}{4}\right\rceil\right) .
$$

It is easy to check that all vectors have length $\sqrt{n_{0}}$, and that by (1) the distance between any two distinct vectors is $\geq \sqrt{n_{0}}$. It follows that (2) is a lower bound on $\tau_{n}$.

\section{Remarks}

(1) Even if we do not know the exact values of $A(n, d, w)$ and $A(n, d)$ mentioned in (2), we can replace them by any available lower bounds, and still obtain a lower bound on the kissing number $\tau_{n}$. There is some freedom in choosing the $n_{\nu}$, which helps to compensate for our ignorance. 
THE ELECTRONiC JOURNAL OF COMBINATORICS 5 (1998), \#R22

(2) A table of lower bounds on $A(n, d)$ has been given by Litsyn [7], extending the table in [9]. A table of lower bounds on $A(n, d, w)$ for $n \leq 28$ is given in [2], but for larger $n$ little is known. A very incomplete table for $n>28$ can be found in [15].

(3) The construction gives a set of points on a sphere with angular separation of $60^{\circ}$. It can obviously be modified to produce spherical codes with other angles.

\section{Examples}

We illustrate the construction by giving new records in dimensions $32,36,40,44,64,80$ and 128. For other examples see [12], and for further details about the codes see [7], [15].

$\mathbf{n}=32$. We take $n_{0}=32, n_{1}=8, n_{2}=2$ and use $A(32,8) \geq 2^{17}$ from [3], $A(32,8,8) \geq 1117$ from the complement of a lexicographic code $\mathcal{C}(32,8,24)$ (cf. [4]), obtaining a kissing number of $A(32,32,32) A(32,8)+A(32,8,8) A(8,2)+A(32,2,2) A(2,1) \geq 1 \cdot 2^{17}+1117 \cdot 2^{7}+\left(\begin{array}{c}32 \\ 2\end{array}\right) \cdot 2^{2}=$ 276,032 .

$\mathbf{n}=36$. Let the 36 coordinates be labeled $(i, j), 0 \leq i, j \leq 5$, and let the symmetric group $S_{6}$ act by $(i, j) \rightarrow\left(i^{\pi}, j^{\sigma(\pi)}\right)$, where $\pi \in S_{6}$ and $\sigma$ is the outer automorphism of $S_{6}$. One can find a set of 17 orbits under the alternating group $A_{6}$, of sizes ranging from 45 to 360 , whose union forms a constant weight code showing that $A(36,8,8) \geq 2385$. We take $n_{0}=32, n_{1}=8, n_{2}=2$ and obtain a kissing number of $A(36,32,32) A(32,8)+A(36,8,8) A(8,2)+A(36,2,2) A(2,1) \geq$ $1 \cdot 2^{17}+2385 \cdot 2^{7}+\left(\begin{array}{c}36 \\ 2\end{array}\right) \cdot 4=438,872$.

An alternative approach can be based on Warren D. Smith's discovery (personal communication, May 1997) that the 2754 minimal vectors of the self-dual length 18 distance 8 code over $\mathbb{F}_{4}[8]$ yields $\tau_{36} \geq 2754 \cdot 2^{7}=352,512$ by changing any even number of signs. By adjoining additional vectors with fractional coordinates R. H. Hardin and N. J. A. Sloane increased this to 386,570 , which held the record until it was overtaken by the present construction. It is quite possible that with better clique-finding the $\mathbb{F}_{4}$ approach will regain the lead.

$\mathbf{n}=40$. We take $n_{0}=40, n_{1}=8, n_{2}=2$, use a lexicographic code for $A(40,8,8)$, and obtain $A(40,40,40) A(40,10)+A(40,8,8) A(8,2)+A(40,2,2) A(2,1) \geq 1 \cdot 589824+3116 \cdot 2^{7}+\left(\begin{array}{c}40 \\ 2\end{array}\right) \cdot 2^{2}=$ 991,792 . 
THE ELECTRONiC JOURNAL OF COMBINATORICS 5 (1998), \#R22

$\mathbf{n}=44 . \quad A(44,44,44) A(44,11)+A(44,8,8) A(8,2)+A(44,2,2) A(2,1) \geq 1 \cdot 2^{21}+6622 \cdot 2^{7}+$ $\left(\begin{array}{c}44 \\ 2\end{array}\right) \cdot 4=2,948,552$.

$\mathbf{n}=48$. In 48 dimensions the three known unimodular extremal unimodular lattices [5], [10] have kissing number 52,416,000. Our present construction gives less than half this value.

$\mathbf{n}=64$. The words of weight 16 in an extended cyclic code $\mathcal{C}(64,16)$ of size $2^{28}$ from [13] show that $A(64,16,16) \geq 30,828$. In this way we obtain a kissing number of $331,737,984$.

$\mathbf{n}=80$. By taking 4 orbits under $L_{2}(79)$ we obtain $A(80,16,16) \geq 143,780$. We take $n_{0}=64$, $n_{1}=16, n_{2}=4, n_{3}=1$ and obtain $\tau \geq 1,368,532,064$.

$\mathbf{n}=\mathbf{1 2 8}$. This is the most dramatic improvement, so we give a little more detail. Our construction uses:

$$
\begin{aligned}
& A(128,128,128) A(128,32) \text { vectors } \pm 1^{128}: \geq 1 \cdot 2^{43} \\
& A(128,32,32) A(32,8) \text { vectors } \pm 2^{32} 0^{96}: \geq 512064 \cdot 2^{17} \\
& A(128,8,8) A(8,2) \text { vectors } \pm 4^{8} 0^{120}: \quad \geq 2704592 \cdot 2^{7} \\
& A(128,2,2) A(2,1) \text { vectors } \pm 8^{2} 0^{126}: \quad \geq \frac{\left(\begin{array}{c}
128 \\
2
\end{array}\right) \cdot 4}{8,863,556,4} \\
& \text { for a total of } \overline{8,863,556,495,104}
\end{aligned}
$$

Here $A(128,32) \geq 2^{43}$ comes from a BCH code [9, p. 267], $A(128,32,32) \geq 512064$ from a union of two orbits under $L_{2}(127), A(32,8) \geq 2^{17}$ from [3], and $A(128,8,8) \geq 2704592$ is obtained by shortening a $\mathcal{C}(129,8,8)$ of size 2883408 formed from the union of 11 orbits of size 262128 under $L_{2}(128)$. The result is more than 40 times that of the Mordell-Weil lattice.

We do not expect any of these new records to survive for long, since our lower bounds for $A(n, d)$ and $A(n, d, w)$ are very weak. However, it is interesting that such a simple construction gives such dramatic improvements over the kissing numbers of the best lattices known. 
THE ELECTRONiC JOURNAL OF COMBINATORICS 5 (1998), \#R22

\section{References}

[1] C. Bachoc and G. Nebe, Extremal lattices of minimum 8 related to the Mathieu group $M_{22}$, J. reine angew. Math. 494 (1998), 129-140.

[2] A. E. Brouwer, J. B. Shearer, N. J. A. Sloane and W. D. Smith, A new table of constant weight codes, IEEE Trans. Inform. Theory 36 (1990), 1334-1380.

[3] Y. Cheng and N. J. A. Sloane, Codes from symmetry groups and a [32,17,8] code, SIAM J. Disc. Math. 2 (1989), 28-37 .

[4] J. H. Conway and N. J. A. Sloane, Lexicographic codes: error-correcting codes from game theory, IEEE Trans. Inform. Theory 32 (1986), 337-348

[5] J. H. Conway and N. J. A. Sloane, Sphere Packings, Lattices and Groups, Springer-Verlag, NY, 3rd edition, 1998.

[6] N. D. Elkies, personal communication, 1998.

[7] S. Litsyn, An updated table of the best binary codes known, in W. C. Huffman and R. A. Brualdi, editors, Handbook of Coding Theory, Elsevier, Amsterdam, 1998, to appear.

[8] F. J. MacWilliams, A. M. Odlyzko, N. J. A. Sloane and H. N. Ward, Self-dual codes over GF(4), J. Combin. Theory, Series A, 25 (1978), 288-318.

[9] F. J. MacWilliams and N. J. A. Sloane, The Theory of Error-Correcting Codes, NorthHolland, Amsterdam, 1977.

[10] G. Nebe, Some cyclo-quaternionic lattices, J. Alg. 199 (1998), 472-498.

[11] G. Nebe, A method of computing the minimum of certain lattices, preprint.

[12] G. Nebe and N. J. A. Sloane, Table of the highest kissing numbers presently known, published electronically at http://www.research.att.com/ njas/lattices/kiss.html.

[13] W. W. Peterson and E. J. Weldon, Jr., Error-Correcting Codes, MIT Press, Cambridge, MA, 2nd ed., 1972

[14] H.-G. Quebbemann, Lattices with theta-functions for $G(\sqrt{2})$ and linear codes, J. Alg. 105 (1987), 443-450

[15] E. M. Rains and N. J. A. Sloane, A table of lower bounds on constant weight codes, published electronically at http://www.research.att.com/ njas/codes/Andw/. 